\title{
Research proposal of a new clinic model for the interpretation of Lateral Elbow Pain: is it time to change?
}

\author{
L. Di Filippo ${ }^{1,2}$, D. Pennella ${ }^{1,3}$, F. Maselli, 4,5 , Arrigoni ${ }^{6,7}$ \\ 1 University of Tor Vergata, Medicine Department, Rome, Italy \\ 2 FisioAnalysis Maedica, Alessandria, Italy \\ 3 Manual Therapy Lab Clinic, Bari, Italy \\ ${ }^{4}$ DINOGMI Department, Genoa University, Genoa, Italy \\ 5 Sovrintendenza Sanitaria Regionale Puglia INAIL Bari, Italy \\ 6 University of Studies of Milan, Milan, Italy \\ 7 ASST Gaetano Pini CTO
}

\author{
CORRESPONDING AUTHOR: \\ Filippo Maselli \\ Genoa University \\ Genoa, Liguria, Italy \\ Phone: +39 3471951139 \\ E- mail: masellifilippo76@gmail.com
}

DOI:

10.32098/mltj.01.2020.07

LEVEL OF EVIDENCE: 5

\section{ACRONIMS}

MSDs: Musculoskeletal disorders; LBP: Low Back Pain; NP: Neck Pain; SP: Shoulder Pain; GP: Groin Pain; LET: Lateral Elbow Tendinopathy; LE: Lateral Epicondylitis; TE: Tennis Elbow; PPT: Pain Pressure Threshold; ERCB: Extensor Carpi Radialis Brevis; RLCL: Radial-Lateral Collateral Ligaments; SMILE: Symptomatic Minor Instabilities of the Lateral Elbow; LEP: Lateral Elbow Pain; I-APPLEp: Integrated approach of Lateral Elbow Pain; RCT: Randomized Clinical Trial; PSR: Psychosocial Risk; PGFS: Pain Grip Free Strength; LS: Load Stress; LC: Load Capacity; MT: Manual Therapy; MWM: Mobilization with Movement; TEx: Therapeutic Exercise; MRI: Magnetic Resonance Image; US: Ultrasuond; NSLEP: Non-specific Lateral Elbow Pain; SLEP: Specific Lateral Elbow Pain

\begin{abstract}
SUMMARY
Background. Lateral Elbow Tendinopathy (aka Tennis Elbow or Lateral Elbow Pain), is characterized by a specific pain located in the lateral epicondyle and referred to the dorsal compartment of the forearm. Manual labour and forceful gripping activities can exacerbate pain, by restricting a patient's ability to work, train and perform simple daily tasks. In the framework of an aetiopathogenic complexity, a multimodal approach based upon the exclusive involvement of the common extensor tendon seems to be rather obsolete. This method does not consider any intra-articular pain resulting from micro-instabilities requiring a different approach together with a detection performed in a limited time span, in order to avoid any impairment of the psychosocial condition following the failure of all the proposed conservative treatments.

Objectives. The primary objective of this debate was to have elaborated a decisional algorithm called Integrated Approach of Lateral Elbow Pain (I-APPLEp), which could allow the detection of a time-optimized plan for a diagnostic, therapeutic framing.

Discussion. Following the above-mentioned research proposal, this clinical approach based upon an evaluative and therapeutic management of patients with LEP allows us to draw some conclusions:

1) using the expression Lateral Elbow Pain (LEP) to refer to this musculoskeletal condition should be preferable, owing to its aetiological and pathophysiological complexity. Moreover, the expression Non-specific Lateral Elbow Pain (NSLEP) in case of suffering of the extra-articular structures (i.e. Tendinopathy) and Specific Lateral Elbow Pain (SLEP) is recommended, within the framework of arthropathy or intra-articular pathology (e.g.: Smile Symptomatic MInor Instabilities of the Lateral Elbpw);2) an early surgical treatment can be a feasible option in the management of articular micro-instabilities, recalcitrant pathologies and psychosocial profiles with a high risk of unfavorable prognosis.

Conclusions. We recommend testing brand-new diagnostic, therapeutic proposals on sampling patients, who must be adequately chosen, in order to estimate the real impact in the short, medium and long term.
\end{abstract}

\section{KEY WORDS}

Lateral elbow tendinopathy; tennis elbow; lateral elbow pain 


\section{BACKGROUNDS}

Musculoskeletal disorders (MSDs) are a common public health problem, especially for the general population. Actually, they play a relevant role in the healthcare systems of the entire world (1). In terms of global impact on the general state of health, these disorders are second only to mental and behavioral diseases, not to mention the serious functional limitations they trigger, which affect individuals' social life dramatically (2). As regards musculoskeletal physical therapy, the modern diagnostic orientation seems keen on classify as "non-specific" the most commons MSDs as Low Back Pain (LBP), Neck Pain (NP), Shoulder Pain (SP) or Groin Pain (GP), which are pretty common among the general population. By doing so, one can implicitly suppose an unknown pathoanatomical origin of this painful disorder, in order to overcome the deadlock of the impossible detection of a pathoanatomical pain generator (e.g. bones, ligaments, capsule, muscles, intervertebral fibrocartilage etc.) with the usual evaluative and diagnostic tools used by clinicians. We are inclined to suggest that this assumption should be applied to further disorders affecting different articulations (elbows included) like Lateral Elbow Tendinopathy (LET), Lateral Epicondylitis (LE) or Tennis Elbow (TE). As far as LET is concerned, this disorder is characterized by a pain located in the lateral epicondyle and referred to the dorsal compartment of the forearm. Pain is exacerbated by manual labour and forceful gripping activities, with a subsequent limitation of the activities of daily living of the patient, as well as his/her working life and training (3). What is more, it is generally considered an extra-articular condition originated from a degeneration of the proximal origin of common extensor tendons (4). Allegedly, this definition does not leave any room for further eventual considerations about possible pain generators, although tendons might not be the main problem, since it is only a step of the multifactorial process involving extra-articular, intra-articular, psychosocial and systemic factors (5). As we stated before, this condition has been described with different expressions such as "lateral elbow tendinopatby" "lateral epicondylalgia", "tennis elbow", "lateral epicondylitis" or "lateral epicondylosis". The common ground among these expressions was the self-evident pain in the extensor carpi radialis brevis muscle (6), which led many authors to use the abbreviation LET in order to detect it promptly. The natural course of this kind of disorder is generally unfavourable, with a low spontaneous remission of its symptoms within a year and an average recurrence within 18 months in 50 percent of cases or within 3-5 years, in 20 percent of cases (7). Furthermore, we are inclined to infer that this musculoskeletal disorder originates from specific alterations of the psychosocial sphere, which affect both diagnosis and prognosis negatively (i.e. low social support, anxiety, depressive symptoms, passive coping as well as negative phrasing) $(8,9)$. A great number of authors have notably pointed out a negative correlation among LET prognosis, catastrophizing and stress (10).

\section{A new model proposed by Coombes et al.}

These data suggest that the apparent simplicity in the detection of a clinical presentation of such disease is counterbalanced by an increased general complexity emerging from the current most followed, widespread model of assessment and treatment shared by professionals specialized in the management of LET. Namely, the above-mentioned model is centered upon the integration of the three basic pillars of the clinical diagnostic framework of patients, according to a multimodal approach $(11,12)$ :

- pathological conditions of tendons: every detected generative process could be regarded as an effect of overuse (i.e. thickening, thinning, tendon injuries, calcification, neovascularization etc.). As a result, the ability of tendons to respond to mechanical stimuli by means of a balance between collagen degradation and synthesis could lead to negative results (13). In addition, the Extensor Carpi Radialis Brevis (ECRB) muscle might be in a disadvantaged mechanical condition due to an increased friction of the tendon insertion, a compression and a cutting force on the epithroclea as well as the presence of an hypo-vascularised area localized in the pre-insertional portion and subsequent histological, macroscopic alterations (14);

- changes in the pain modulation system: increment of both P-substance and glutamate (mediators of the neurogenic pain) (15), presence of latent trigger points in the contralateral limb with subsequent abnormal sensitivity to pain (hyperalgesia) and Pain Pressure Threshold (PPT) reduction. All these factors could lead to a central sensitization, especially in case of LET lasting more than three months (16);

- sensory processing disorders: decrement of grip strength; weakness of specific muscular groups such as flexors, wrist extensor muscles and rotator cuffs (17); alterations of motor control (e.g. delayed activation timing of ECRB in case of a tennis player performing backhand), reduction of the endurance of the back-extensor muscles, impossible handgrip task performance and decreasing reaction time (18). These disorders can also involve contralateral limbs. On the basis of the above-mentioned model, many authors like Coombes, Bisset and Vincenzino elaborated a treatment algorithm as they suggested managing this kind of disorders in line with definable prognostic factors such as tendon condition, severity and disability of tendon pathology, associated pain, central sensitivity, sensory processing disorders and work conditions (19).

Muscles, Ligaments and Tendons Journal 2020;10 (1) 


\section{The model of Coombes et al. and its limits}

Notwithstanding that, we believe that this model shows some weak points:

- both education and information concerning the handling of the loads and the ergonomic approaches for the improvement of workplace and in sport system optimization seem to be rather reductive strategies, in case of diseases that are not self-limiting;

- although negative prognostic factors do encompass psychosocial conditions, very few clinical elements can prove its presence, along with its relative risk and its impact on a treatment programme;

- progression in therapy depends upon time. Both instrumental diagnostic investigations and surgical consults are recommended only afterwards, in case of recalcitrant pathologies, most likely when the clinical picture is irretrievably damaged.

\section{New pathoanatomical acquisitions}

The focus on a likely correlation between recalcitrant pathologies and cases of articular micro-instability has increased the attention on this topic over the last years, especially in case of patients showing a high predisposition for pain perception and a negative outcome after testing articular macro-instability. These patients have shown an increased incidence of intra-articular reactions that can be easily related to a pathological laxity, which can be defined "elbow lateral (micro) instability", especially in case of recalcitrant elbow lateral pain, even though it was originally defined as LET (20). Further authors $(20,21)$ have also highlighted signs of intra-articular pain, after arthroscopy (i.e. synovitis, radio-ulnar ballottement, radiocapitellar chondromalachia combined with a pathological laxity of both radial-lateral collateral ligaments (RLCL) and orbicular ligaments. This clinical picture could be a clue of the presence of symptomatic minor instabilities of the lateral elbow (aka SMILE). In some cases, a common extensor tendinopathy could follow RLCL elongations triggered by repeated stress in case of pronation of forefoot varus, which might imply chronic pain (20) (figure 2). Nevertheless, rarely have patholaxity and minor instability been considered as the sources of LEP. Therefore, this disorder might originate from an overuse and/or exposure to repetitive loads, which could provoke tissue patholaxity, secondary chondropathy and persistent pain involving osteochondral section. This conclusion goes beyond the previous assumption on the exclusive involvement of the tendon-muscular section in this disorder. In view of the above, elbow painful syndromes could be one of those musculoskeletal disorders that are influenced by anatomic, psycho-social and chronological factors: these elements can seriously reduce the possibility of providing a reliable diagnosis, whilst relegating patients to deep concern, low self-esteem, social isolation and trust loss (22).

\section{A problem of terminology}

Despite the shared consensus on the clinical characteristics of this pathology, the incidental analysis of the inclusive parameters of some studies concerning this kind of musculoskeletal disorders and their effects on psychosocial life $(8,9,23)$ shows a surprisingly high heterogeneity of opinions about their definitions: LET, Tendinopathy, Epicondylosis, Epicondylitis, TE. Thus, the most generic definition of Lateral Elbow Pain (LEP) could be preferable, as the literature suggests. It could be possible to overcome the idea that only a structure should elicit patients' symptomatology. On the contrary, one should recognize the great aetiological, pathophysiological complexity of LEP, by emphasizing the relevance of psycho-social factors, which are inevitably related to this disorder, as it has already been proven in case of further anatomic districts (i.e. shoulder, neck and low back spine) (24). If we consider the aetiopathogenic complexity of LEP, we must subsequently rule out a multimodal approach based upon the exclusive involvement of the common extensor tendon, for it is obsolete and unable to detect possible intra-articular pain related to minor instabilities. Yet, this clinical picture should require a wholly different approach; above all, it should be assessed according to a strictly reduced quantity of time, in order to avoid any impairment of the psychosocial condition ensued from the failure of the proposed conservative treatments (8). This is the reason why we have elaborated a decisional algorithm called I-APPLEp (Integrated approach of Lateral Elbow Pain)" (figure 1), for patients suffering from LEP, which could allow the identification of a reliable time-optimized diagnostic-therapeutic framing. The aim of this algorithm is not to consider the set of conservative, surgical approaches in a progression that follows the implemented therapy in case of previous failed treatment. On the contrary, its objective is to refer them to the specific patient, the characteristics of his/her symptoms the aetiological hypothesis of his/ her pain and the scrutiny of the share of responsibility of those pain-generator structures potentially involved in the patient's symptomatology.

\section{Scientific evidence for the psychosocial sphere}

Various studies in the literature have underscored increased level of anxiety in patients suffering from LEP from third month onwards. Anxiety is presumably a consequence of the following factors: 


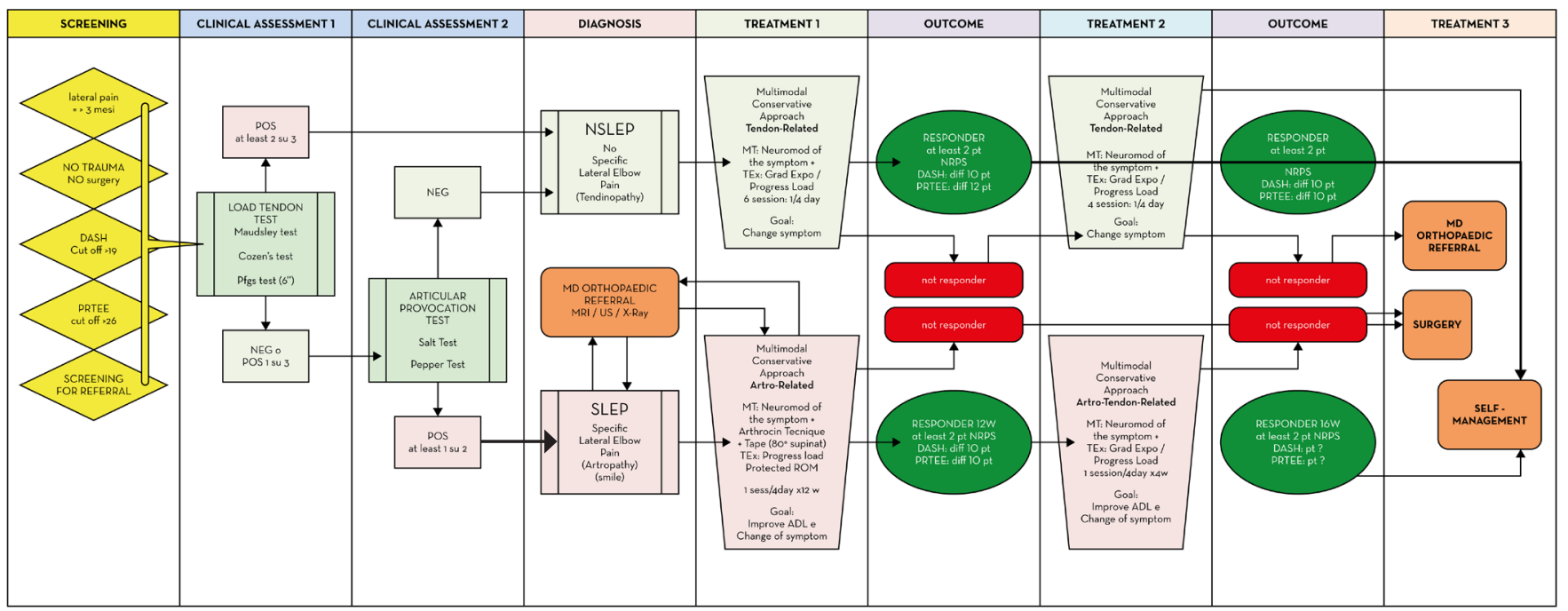

Figure 1. Integrated Approach of Lateral Elbow Pain (I-APPLEp) - a decision algorithm for a detection of a time-optimized plan for management of lateral elbow pain.

- increase of chemical mediators of neurogenic pain, PPT decrement, presence of trigger points and bilateral sensitive-motor alterations, which might be a symptom of the onset of central sensitiveness (25);

- articular minor instability: this condition requires a longer management process along with a different diagnostic-therapeutic pathway $(8,19)$. This procedure might

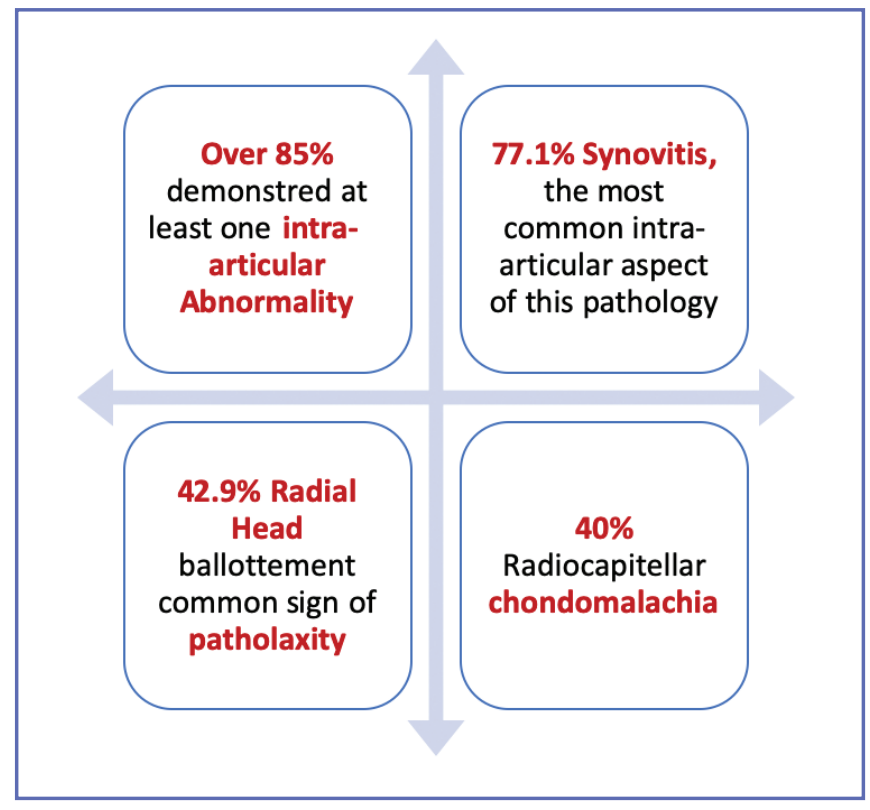

Figure 2. Intra-articular abnormal finding detected in elbow lateral pain with arthroscopy be justified especially in case of diseases for which one can observe a negative reaction to previous treatments;

- psychosocial factors (low social support, poor control at work, low job satisfaction, depressive symptoms) $(8,10)$ are common in most of the patients who have suffered from LEP for more than three months.

Considering the above, we believe that it is of the utmost importance that patients suffering from LEP be examined also by means of indirect assessment questionnaires of psychosocial factors. For instance, DASH (8) is a test that help link these disorders with depression, anxiety and pain catastrophizing; besides, PRTEE (8) combines these diseases with poor life quality and sleep disorders (26). Thanks to these rating scales, it is possible to find out cut-off values, i.e. $\mathrm{DASH}>19$ and PRTEE $>26$; values over these thresholds suggest that psychosocial factors may be involved in this kind of disorder, do the symptom detected persist for more than 3 months and providing it is related to specific professions.

\section{Scientific evidence for the therapeutic use of braces} We have considered and analyzed the full texts of 16 articles: among them, we considered three Randomized Clinical Trial (RCT) on the effectiveness of Taping in case of Elbow Tendinopathies (27-29), four RCT on epicondylalgia (30-32) and one more article on articular limitation of a safe elbow (33). Unfortunately, the accumulated evidence on the effectiveness of these restraining techniques in a typi- 
cal elbow musculoskeletal disease is poor and contradictory: orthotic devices are clearly no scientific basis, whereas the use of an orthopedic cast must be discouraged. Taping/ bandaging is also not effective; however, some authors have identified effects on improving the strength of wrist extension, grip strength and perceived pain in the short term (34): indeed, in line with the most recent studies, it seems mandatory to reconsider this technique within a multimodal approach, by using new selection criteria for patients, which can clinically distinguish LEP from SMILE, provided that it is specified whether it is a rigid tape or a more elastic one. As shown by the finding of the above-mentioned analysis, it is possible to select the methods that can be followed during LEP management, with regard to a specific clinical context.

\section{DISCUSSION}

The discrepancy between linear processes of biological repair of living tissues and non-linear processes of pain resolution is, by far, the most relevant gap among LEP assessment, history and treatment. It is a fact that patients suffering from LEP show signs of tissue sufferance, via structural imaging (35); yet, there is no connection whatsoever among these signs, the severity of illness and the perception of pain. Further factors are implied in this case (36). However, ruling out any of these elements would be impossible; on the contrary, they should all be integrated in a global assessment process. Studies on arthroscopy show that $85 \%$ of patients suffering from LEP reveal intra-articular anomalies, 77.1\% report synovitis, $42.9 \%$ radio-ulnar ballottement, and $40 \%$ suffer from radiocapitellar chondromalachia (figure 2). It is important to notice that both ballottement and chondropathies are less frequent, although intra-articular anomalies and synovities are the common ground of almost all patients. This observation leads us to support the hypothesis that, in some cases, the complexity of the joint impairment called SMILE might be the principal source of pain. The clinical situation of these patients is worsened by a further overload of the common extensor tendon, which acts like a dynamic stabilizer against stress caused by forearm pronation and varus. Especially during daily activities, maximum articular stability is strictly requested $(37,38)$. In the light of this rather complex clinical condition, one can notice that the natural course of LEP is highly unfavorable: only $13 \%$ of the examined patients show a spontaneous remission of symptoms after 3 months, and 34\% of them get successfully recovered after 12 months. In both cases, low remission rates are linked to low social support and passive coping (5): these factors should not be underestimated during the assessment and the treatment of patients with LEP. Actually, musculoskeletal pain lasting more than 3 months shows meaningful connections with both anxiety and depression, which are often related to financial, social, psychological and occupational problems (39). As proven by some authors, all these factors may seriously affect the management of patients with LEP, mostly because they force to find the best possible pathway to reduce time and avoid the development of psychosocial impairment resulting from chronic symptoms $(8,9,40)$. Accordingly, several studies have shown a remarkable correlation between LEP and the presence of anxiety and depression $(8,23,39,41)$, including kinesiophobia, pain catastrophizing (42), low job satisfaction, low job support $(41,43,44)$, bad health conditions (39), poor quality of life and sleep disorders (21). An integration of the currently most implemented clinical model will be urgent as long as anatomical impairments play a key role in the evaluation and the management of LEP. Indeed, they could act as pain generators, together with psychosocial factors that cannot determine any comorbidity, risk factors, prognostic factors or obstacles in therapy. We are fully persuaded that this model must be taken into account in order to optimize time planning by avoiding chronic symptoms and potential risks of recalcitrant or treatment-resistant disease $(21,45)$. I-APPLEp is an algorithm created to this purpose: as shown in figure 1, it can be divided into 3 parts, i.e. initial screening, clinical assessment and therapeutic procedure. The initial screening has a double function: firstly, it is performed to detect either red flags or yellow flags, in order to verify the clinical conditions that request hospitalization or another specialized physician; secondly, it quantifies the eventual psychosocial risk of the patient. The detection of the so-called red flags - a rather complicated procedure entailing reasoning and decisions - is performed through an accurate anamnesis and a peculiar physical examination; DASH $(23,26,41)$ and PRTEE $(41,42,44)$ are the most commonly used self-administered scales for the identification of the perceived disability and can be utilized to quantify Psychosocial Risk (PSR). After analyzing all the studies carried out in the literature, we have finally detected some cut-off values emerging from both DASH and PRTEE, which may elucidate the significant role played by psychosocial factors. Did these values go beyond the threshold, specific caution should be paid to the source of this disorder as well as the performance of the pain itself. Patients must be reassured, and special attention must be paid on the strengthening and the importance of a therapeutic relationship and cooperation between patients and physicians. This approach should be recommended, regardless of the undertaken therapeutic procedure, especially if disorders has been lasting for less than 3 months. The aim is to avoid both complex pain and changes in the pain modulation system. The second step in the assessment procedure aims to find out what anatomic 
structure is responsible for patient's painful symptomatology: in other words, the objective is to suggest what kind of treatment is most likely to be effective for the targeted structure. We are well aware that there is no such thing as a singular, unique anatomic structure that can be seen as a pain generator, considering the complexity of the studied disease, which encompasses intra-articular pain, extra-articular pain and systemic factors (46). It is undeniable that the effectiveness of manual therapy along with specific training in case of chronic/persistent LEP is widely reported in the literature (47-49). Nevertheless, the parameters for the exclusion of the studies concerning LEP treatment do not take into account the possibility to gather patients together according to the kind of tissue most involved in the disease. Up to now, the Myotendinous Junction has been considered as the one and only source of this kind of disorder (50). Yet, there is a large number of studies proving the presence of countless intra-articular lesions associated with LEP (e.g. plica, lesions of the internal capsule, synovitis, ulnar head or radiocapitellar erosion/chondromalachia and laxity of the radial laxity of the radial component of the radial-lateral collateral ligament (RLCL) (51-55). In this phase of the approach, the BC Physical Therapy Tendinopathy Task Force recommends specific tests like Maudsley's Test, Cozen's Test and Pain Grip Free Strength (PGFS), whose purpose is to elicit a load stress (LS) of the myotendinous structure and eventually assess the mechanical response (i.e. Load Capacity, LC). In case at least 2 out of 3 tests are positive, we suppose an altered relationship LS/LC of the myotendinous structure of the Extensor Tendon compartment of the wrist, which can be labelled as Functional Tendinopathy. The procedure goes on with a clinical trial in order to reduce the symptom. The trial consists of a six-session multimodal conservative approach (once every four days) and encompasses:
- a Manual Therapy (MT) to stimulate a neuromodulation of the symptom through myofascial release techniques, Dry Needling, Mobilization with Movement (MWM), for further details see table I;

- therapeutic Exercise (TE): the aim is to improve the relationship between LS and LC, by starting with isometric contractions and progressing with eccentric and/or concentric contractions with pronation and supination of the elbow, instead of wrist flexion-extension.

In case of no response at the end of the clinical trial, patients must be submitted for further clinical and diagnostic assessment (including $\mathrm{X}$-ray and Magnetic Resonance Image (MRI), wherever possible) to an orthopaedic surgeon who shares the same view on LEP. After that, a 12-16-week conservative approach (1 session every four days) will help reduce symptoms and improve Activity Day Living, by performing the treatments below:

- MT: the objective of this procedure is to attempt symptom neuromodulation through myofascial release techniques, Dry Needling, MWM and Arthrokinematic Techniques;

- TE: once again, the purpose is to try to improve the relationship LS/LC by performing high intensity contractions and Range of Motion (ROM) integrated with functional bandage, by using both Flexion-Extension movements and Elbow Pronation/Rehabilitation.

Were the patient still labelled as 'NO RESPONDER', surgery may be considered, in accordance with the specialist physician's advice. During the assessment-decision phase, in case one in three tests is positive, or at least two in three tests are negative, two further tests are administrated, provided that Red Flags or cervical related causes are ruled out. The purpose of the aforementioned tests is to study the possible

Table I. Definitions of Manual Therapy (MT) and Mobilization with Movement.

\begin{tabular}{ll}
\hline Name of the group of techniques & Description \\
\hline Manual Therapy (MT) & It may be defined as "the use of hands to apply a force with a therapeutic intent" (65). \\
Widely used MTs are spinal manipulative therapy (SMT), peripheral manipulative \\
therapy, joint mobilization and/or deep tissue massage, soft tissue mobilizzation, \\
miofascial release, etc. It has been shown to relieve symptoms in various musculoskeletal \\
conditions (66). It includes myofascial treatment and manual mobilizations of the elbow \\
and wrist joint, with a specific target centered on myofascial and articular lesions found in \\
patients with lateral elbow pain.
\end{tabular}


involvement of intra-articular structures in patients suffering from recalcitrant LEP (27), after the initial comparison with arthroscopy:

- supination and Antero-Lateral pain test (SALT) has revealed a high sensitivity and a low specificity. It is the most accurate method to follow in case of synovitis;

- posterior Elbow Pain by Palpation-Extension of the Radiocapitellar Joint (PEPPER): this test has gained positive outcomes in the detection of Radiocapitellar Chondromalachia.

Patients suffering with LEP usually belong to two different subcategories, depending on the predominant component of the source of pain:

- NSLEP: Non-specific Lateral Elbow pain, every time Tendinopathy is predominant;

- SLEP: Specific lateral elbow pain, for predominant intra-articular component (SMILE o arthropathy).

Here are the main differences, in case of a predominant intra-articular component (SLEP):

- longer-lasting conservative multimodal approach with regard to (NSLEP): 1 session every 4 days for 12-16 weeks;

- insertion of an articular protection factor (humeroradial and conoid ligament) or physical training performed with limited articular excursion exercises, functional bandage (braces/tape) in order to stimulate cutaneous neurotransmitter receptors. The effectiveness of this technique is widely recognized as well as the sensation of betterment, increased stability and enhanced grip strength (32);

- the instrumental diagnostic confirmation of the anatomic condition via MRI is requested to examine both soft tissues and connective tissues like articular cartilage and/ or capsule (56-58).

Current evidence indicates that intralesional infiltration of corticosteroids should be rejected, since the benefits of this pharmacological treatment after six months are blithely comparable to placebo. Thus, there are at least two substantiated reasons to discourage this approach: firstly, it delays intra-articular tissues repair. Secondly, it predisposes patients to both an arguably worsened osteoarticular condition and a time-dependent psychosocial impairment. Unless patients suffering from SLEP improved their health condition significantly after 16 weeks of conservative treatment, they would be forced to undergo surgery. In this case, a surgical intervention could reduce anatomic impairment joined to a possible minor instability (i.e. SMILE) and an arthropathic frame $(51,54,55,59)$; furthermore, it to avoids complications always occurring when symptoms become chronic.
If we depicted LEP in this way, an increased number of patients could allegedly benefit from surgery $(50,60-62)$. The currently estimated percentage is $5-10 \%$ (63), bearing in mind that there is also a low incidence of complications and iatrogenic damage (e.g. elbow extension restriction, elbow flexion restriction and/or strength lost) linked to the specific surgical practices that patients can always tolerate (64). These complications can be seen as adverse effects and related either to a failed surgical intervention or, even more often, to an inappropriate physical therapy (64).

\section{CONCLUSIONS}

On the one hand, we firmly believe that framing Elbow Lateral Epicondylitis as a mere nosological entity contradicts evidence. On the other hand, it is preferable to use a multi-factorial diagnostic-therapeutic approach that consider the importance of local intra-articular, extra-articular/ tendon related, systemic and psychological factors, which seem to help detect the level of impairment of psycho-social factors, as observed in many other musculoskeletal conditions (i.e. non-specific low back pain, non-specific neck pain and shoulder pain syndrome). We propose I-APPLEp, a brand-new approach based upon assessment and treatment, which is built upon clinical reasoning and decision-making. This approach is perfectly suited to this purpose, and it is in line with the characteristics of the so-called Bio-Psychosocial model. This is an attempt to provide clinicians with a useful operative tool in order to meet the requests of the patients in the most effective and efficient way. By adopting I-APPLEp, one can avoid treatment resistance which underlies the worsening of the clinical framework; what is more, it prevents/limits psychosocial alterations (e.g. anxiety, depression, negative phrasing, passive coping etc.), which play a crucial role in the compliance and healing of patients. According to this clinical approach, both assessment and treatment of patients suffering from LEP highlight two important topics:

- the expression (LEP) is able to describe more accurately this musculoskeletal condition, given its aetiological and pathophysiological complexity; besides, we suggest using the expression Non-specific Lateral Elbow Pain in case of Tendinopathy and SLEP, within the frame of arthropathy and intra-articular disease (e.g. SMILE);

- precocious surgical treatment should be considered only in case of recalcitrant disease, with high risk of unfavorable prognosis.

Regardless of this fact, a number of critical issues regarding this approach remain to be addressed: 
- as far as we know, no previous study has strongly supported specific DASH values, whose cut-off has been detected with the help of the algorithm called I-APPLEp in 19 different points. These studies address severe clinical pictures and unfavorable prognosis of patients suffering from SLEP for more than 3 months. Questions concerning patients suffering from this pain for less than 3 months remain unanswered, so far;

- the recommended tests proposed in this study has been implemented on a tiny sample, in spite of the reliable diagnostic values.

\section{REFERENCES}

1. Wiitavaara B, Fahlström M, Djupsjöbacka M. Prevalence, diagnostics and management of musculoskeletal disorders in primary health care in Sweden - an investigation of 2000 randomly selected patient records. J Eval Clin Pract. 2017;23(2):325-332.

2. Piano L, Maselli F, Viceconti A, Gianola S, Ciuro A. Direct access to physical therapy for the patient with musculoskeletal disorders, a literature review. J Phys Ther Sci. 2017;29(8):14631471.

3. Longo UG, Franceschetti E, Rizzello G, Petrillo S, Denaro V. Elbow tendinopathy. Muscles Ligaments Tendons J. 2012;2(2):115-120.

4. Bisset LM, Vicenzino B. Physiotherapy management of lateral epicondylalgia. J Physiother. 2015;61(4):174-181.

5. Katz J, Rosenbloom BN, Fashler S. Chronic Pain, Psychopathology, and DSM-5 Somatic Symptom Disorder. Can J Psychiatry. 2015;60(4):160-167.

6. Sims SEG, Miller K, Elfar JC, Hammert WC. Non-surgical treatment of lateral epicondylitis: a systematic review of randomized controlled trials. Hand (N Y). 2014;9(4):419-446.

7. Smidt N, Lewis M, VAN DER Windt DAWM, Hay EM, Bouter LM, Croft P. Lateral epicondylitis in general practice: course and prognostic indicators of outcome. I Rheumatol. 2006;33(10):2053-2059.

8. Das De S, Vranceanu A-M, Ring DC. Contribution of kinesophobia and catastrophic thinking to upper-extremity-specific disability. J Bone Joint Surg Am. 2013;95(1):76-81.

9. Lee DO, Gong HS, Kim JH, Rhee SH, Lee YH, Baek GH. The relationship between positive or negative phrasing and patients' coping with lateral epicondylitis. J Shoulder Elbow Surg. 2014;23(4):567-572.

10. Mallows A, Debenham J, Walker T, Littlewood C. Association of psychological variables and outcome in tendinopathy: a systematic review. Br J Sports Med. 2017;51(9):743-748.

11. Coombes BK, Bisset L, Vicenzino B. Management of Lateral Elbow Tendinopathy: One Size Does Not Fit All. J Orthop Sports Phys Ther. 2015;45(11):938-949.

12. Day JM, Lucado AM, Uhl TL. A comprehensive rehabilitation program for treating lateral elbow tendinopathy. Int J Sports Phys Ther. 2019;14(5):818-829.
Given the complexity of the diagnostic picture of this condition and the failure of the related therapeutic approach, a more accurate test is required in order to analyses a more adequate number of patients and assess the real impact of this innovative treatment in the short, medium and long term. This work was conducted following "Basic principles and recommendations in clinical and field science research: 2018 update" trying to agree, as much as possible, to the contents and issues discussed in this official editorial (70).

\section{CONFLICT OF INTERESTS}

The authors declare that they have no conflict of interests.

13. Ljung BO, Forsgren S, Fridén J. Substance P and calcitonin gene-related peptide expression at the extensor carpi radialis brevis muscle origin: implications for the etiology of tennis elbow. J Orthop Res. 1999;17(4):554-559.

14. Wright A. Recent concepts in the neurophysiology of pain. Man Ther. 1999;4(4):196-202.

15. Alizadehkhaiyat O, Fisher AC, Kemp GJ, Vishwanathan K, Frostick SP. Upper limb muscle imbalance in tennis elbow: a functional and electromyographic assessment. J Orthop Res. 2007;25(12):1651-1657.

16. Kelley JD, Lombardo SJ, Pink M, Perry J, Giangarra CE. Electromyographic and cinematographic analysis of elbow function in tennis players with lateral epicondylitis. Am J Sports Med. 1994;22(3):359-363.

17. Rojas M, Mañanas MA, Muller B, Chaler J. Activation of forearm muscles for wrist extension in patients affected by lateral epicondylitis. Conf Proc IEEE Eng Med Biol Soc. 2007;2007:4858-4861.

18. Vicenzino B, Paungmali A, Buratowski S, Wright A. Specific manipulative therapy treatment for chronic lateral epicondylalgia produces uniquely characteristic hypoalgesia. Man Ther. 2001;6(4):205-212.

19. Kwak SH, Lee S-J, Jeong HS, Do MU, Suh KT. Subtle elbow instability associated with lateral epicondylitis. BMC Musculoskelet Disord. 2018;19(1):136.

20. Arrigoni P, Cucchi D, D’Ambrosi R, Butt U, Safran MR, Denard P, Randelli P. Intra-articular findings in symptomatic minor instability of the lateral elbow (SMILE). Knee Surg Sports Traumatol Arthrosc. 2017 Jul;25(7):2255-2263. Epub 2017 Mar 24. PubMed PMID: 28341879.

21. Kniesel B, Huth J, Bauer G, Mauch F. Systematic diagnosis and therapy of lateral elbow pain with emphasis on elbow instability. Arch Orthop Trauma Surg. 2014;134(12):1641-1647.

22. Bot S, van der Waal JM, Terwee C, van der Windt DAWM, Bouter L, Dekker J. Course and prognosis of elbow complaints: a cohort study in general practice. Ann Rheum Dis. 2005;64(9):1331-1336.

23. Ring D, Kadzielski J, Fabian L, Zurakowski D, Malhotra LR, Jupiter JB. Self-reported upper extremity health 
status correlates with depression. J Bone Joint Surg Am. 2006;88(9):1983-1988.

24. Maher C, Underwood M, Buchbinder R. Non-specific low back pain. Lancet. 2017;389(10070):736-747.

25. Coombes BK, Bisset L, Vicenzino B. A new integrative model of lateral epicondylalgia. Br J Sports Med. 2009;43(4):252-258.

26. Coombes BK, Bisset L, Vicenzino B. Thermal hyperalgesia distinguishes those with severe pain and disability in unilateral lateral epicondylalgia. Clin J Pain. 2012;28(7):595-601.

27. Au IPH, Fan PCP, Lee WY, et al. Effects of Kinesio tape in individuals with lateral epicondylitis: A deceptive crossover trial. Physiother Theory Pract. 2017;33(12):914-919.

28. Stratford P, Levy D. Assessing Valid Change over Time in Patients with Lateral Epicondylitis at the Elbow. Clinical Journal of Sport Medicine. 1994;4(2):88-91.

29. Shamsoddini A, Hollisaz MT. Effects of Taping on Pain, Grip Strength and Wrist Extension Force in Patients with Tennis Elbow. Trauma Mon. 2013;18(2):71-74.

30. Vicenzino B, Brooksbank J, Minto J, Offord S, Paungmali A. Initial effects of elbow taping on pain-free grip strength and pressure pain threshold. J Orthop Sports Phys Ther. 2003:33(7):400-407.

31. Jafarian FS, Demneh ES, Tyson SF. The immediate effect of orthotic management on grip strength of patients with lateral epicondylosis. J Orthop Sports Phys Ther. 2009;39(6):484-489.

32. Bisset LM, Collins NJ, Offord SS. Immediate effects of 2 types of braces on pain and grip strength in people with lateral epicondylalgia: a randomized controlled trial. $J$ Orthop Sports Phys Ther. 2014;44(2):120-128.

33. Altan L, Kanat E. Conservative treatment of lateral epicondylitis: comparison of two different orthotic devices. Clin Rheumatol. 2008;27(8):1015-1019.

34. Shamsoddini A, Hollisaz MT. Effects of Taping on Pain, Grip Strength and Wrist Extension Force in Patients with Tennis Elbow. Trauma Mon. 2013;18(2):71-74.

35. Fedorczyk JM. Tendinopathies of the elbow, wrist, and hand: histopathology and clinical considerations. I Hand Ther. 2012;25(2):191-200; quiz 201.

36. Moseley GL. A pain neuromatrix approach to patients with chronic pain. Man Ther. 2003;8(3):130-140.

37. Arrigoni P, Cucchi D, Menon A, Randelli P. It's time to change perspective! New diagnostic tools for lateral elbow pain. Musculoskelet Surg. 2017;101(Suppl 2):175-179.

38. Bot S, van der Waal JM, Terwee C, van der Windt DAWM, Bouter L, Dekker J. Course and prognosis of elbow complaints: a cohort study in general practice. Ann Rheum Dis. 2005;64(9):1331-1336.

39. Alizadehkhaiyat O, Fisher AC, Kemp GJ, Frostick SP. Pain, functional disability, and psychologic status in tennis elbow. Clin J Pain. 2007;23(6):482-489.

40. Thiese MS, Hegmann KT, Kapellusch J, et al. Psychosocial Factors Related to Lateral and Medial Epicondylitis: Results From Pooled Study Analyses. J Occup Environ Med. 2016;58(6):588-593

41. Aben A, De Wilde L, Hollevoet N, et al. Tennis elbow: associated psychological factors. I Shoulder Elbow Surg. 2018;27(3):387-392.
42. Haahr JP, Andersen JH. Physical and psychosocial risk factors for lateral epicondylitis: a population based case-referent study. Occup Environ Med. 2003;60(5):322-329.

43. Bugajska J, Żołnierczyk-Zreda D, Jędryka-Góral A, et al. Psychological factors at work and musculoskeletal disorders: a one year prospective study. Rheumatol Int. 2013;33(12):29752983.

44. Berglund KM, Persson BH, Denison E. Prevalence of pain and dysfunction in the cervical and thoracic spine in persons with and without lateral elbow pain. Man Ther. 2008;13(4):295-299.

45. Maffulli G, Hemmings S, Maffulli N. Assessment of the Effectiveness of Extracorporeal Shock Wave Therapy (ESWT) For Soft Tissue Injuries (ASSERT): An Online Database Protocol. Transl Med UniSa. 2014;10:46-51.

46. Antuna SA, O’Driscoll SW. Snapping plicae associated with radiocapitellar chondromalacia. Artbroscopy. 2001;17(5):491495.

47. Valdes K, LaStayo P. The value of provocative tests for the wrist and elbow: a literature review. J Hand Ther. 2013;26(1):32-42; quiz 43.

48. Faculty of physiotherapy, Sri Ramachandra University, Chennai, Tamil Nadu, India., Saroja G, Aseer P AL, Reader in physiotherapy, Sri Ramachandra University, Chennai, Tamil Nadu, India., P M VS, Professor \& Head in Radiology, Sri Ramachandra University, Chennai, Tamil Nadu, India. Diagnostic accuracy of provocative tests in lateral epicondylitis. IJPR 2014;2(6):815-823.

49. Vicenzino B. Physiotherapy for tennis elbow. Evid Based Med. 2007;12(2):37-38.

50. Taylor SA, Hannafin JA. Evaluation and management of elbow tendinopathy. Sports Health. 2012;4(5):384-393.

51. Wada T, Moriya T, Iba K, et al. Functional outcomes after arthroscopic treatment of lateral epicondylitis. J Orthop Sci. 2009;14(2):167-174.

52. Jeon I-H, Chun J-M, Lee C-S, et al. Zona conoidea of the elbow: another articulation between the radial head and lateral trochlear of the elbow. J Bone Joint Surg Br. 2012;94(4):517522.

53. Baker CL, Murphy KP, Gottlob CA, Curd DT. Arthroscopic classification and treatment of lateral epicondylitis: two-year clinical results. J Shoulder Elbow Surg. 2000;9(6):475-482.

54. Rajeev A, Pooley J. Arthroscopic resection of humeroradial synovial plica for persistent lateral elbow pain. J Orthop Surg (Hong Kong). 2015;23(1):11-14.

55. Kim DH, Gambardella RA, Elattrache NS, Yocum LA, Jobe FW. Arthroscopic treatment of posterolateral elbow impingement from lateral synovial plicae in throwing athletes and golfers. Am J Sports Med. 2006;34(3):438-444.

56. Magee T. Accuracy of 3-T MR arthrography versus conventional 3-T MRI of elbow tendons and ligaments compared with surgery. AJR Am J Roentgenol. 2015;204(1):W70-75.

57. Kijowski R, Tuite M, Sanford M. Magnetic resonance imaging of the elbow. Part II: Abnormalities of the ligaments, tendons, and nerves. Skeletal Radiol. 2005;34(1):1-18.

58. Dewan AK, Chhabra AB, Khanna AJ, Anderson MW, Brunton LM. MRI of the elbow: techniques and spectrum of disease: AAOS exhibit selection. J Bone Joint Surg Am. 2013;95(14):e99 $1-13$. 
59. Arthroscopic managementof miscellaneous elbow disorders - ScienceDirect. https://www.sciencedirect.com/science/article/abs/pii/S1060187298800336. Accessed January 22, 2020.

60. Arrigoni P, Cucchi D, D’Ambrosi R, Menon A, Aliprandi A, Randelli P. Arthroscopic R-LCL plication for symptomatic minor instability of the lateral elbow (SMILE). Knee Surg Sports Traumatol Arthrosc. 2017;25(7):2264-2270.

61. Müller A, Spies CK, Unglaub F, Bruckner T, Pötzl W. [Chronic lateral epicondylitis: The Nirschl procedure]. Oper Orthop Traumatol. 2015;27(6):525-535.

62. Phorkhar T, Chanlalit C. Functional Results in Arthroscopic Treatment in Patients with Chronic Lateral Elbow Pain. J Med Assoc Thai. 2015;98 Suppl 10:S130-134.

63. El Hajj F, Hoteit M, Ouaknine M. Elbow arthroscopy: An alternative to anteromedial portals. Orthopaedics E Traumatology: Surgery \& Research. 2015;101(4):411-414.

64. Leong NL, Cohen JR, Lord E, Wang JC, McAllister DR, Petrigliano FA. Demographic Trends and Complication Rates in Arthroscopic Elbow Surgery. Arthroscopy. 2015;31(10):19281932.
65. Smith AR. Manual therapy: the historical, current, and future role in the treatment of pain. ScientificWorldJournal. 2007;7:109-120.

66. Lorås $\mathrm{H}$, Østerås $\mathrm{B}$, Torstensen TA, Østerås H. Medical Exercise Therapy for Treating Musculoskeletal Pain: A Narrative Review of Results from Randomized Controlled Trials with a Theoretical Perspective. Physiother Res Int. 2015;20(3):182190.

67. Kisner C, Colby LA, Borstad J. Therapeutic Exercise: Foundations and Techniques. F.A. Davis; 2017.

68. Manual Therapy: "NAGS", "SNAGS", "MWMS" Etc - Brian R. Mulligan -

69. Hing W, Bigelow R, Bremner T. Mulligan's Mobilization with Movement: A Systematic Review. Journal of Manual E Manipulative Therapy. 2009;17(2):39E-66E.

70. Padulo J, Oliva F, Frizziero A, Maffulli N. Muscles, Ligaments and Tendons Journal - Basic principles and recommendations in clinical and field Science Research: 2018 update. Muscle, Ligaments and Tendons Journal. 2018; 8(3): $305-307$. 